\title{
Polymeric Nanocarriers with Luminescent Colloidal Nanoplatelets as Hydrophilic and Non-Toxic Two-Photon Bioimaging Agents
}

\author{
Katarzyna Celina Nawrot (iD) \\ Jan Kazimierz Zareba (D) \\ Monika Toporkiewicz $\mathbb{D D}^{2}$ \\ Grzegorz Chodaczek (D) ${ }^{2}$ \\ Dominika Wawrzynczyk (I) \\ Julita Kulbacka $\mathbb{D}^{3}$ \\ Urszula Bazylinska $\mathbb{D D}^{4}$ \\ Marcin Nyk (D)
}

\begin{abstract}
'Advanced Materials Engineering and Modelling Group, Faculty of Chemistry, Wroclaw University of Science and Technology, Wroclaw, 50-370, Poland; ${ }^{2}$ tukasiewicz Research Network - PORT Polish Center for Technology Development, Wroclaw, 54-066, Poland; ${ }^{3}$ Department of Molecular and Cellular Biology, Faculty of Pharmacy, Wroclaw Medical University, Wroclaw, 50-556, Poland; ${ }^{4}$ Department of Physical and Quantum Chemistry, Faculty of Chemistry, Wroclaw University of Science and Technology, Wroclaw, 50-370, Poland
\end{abstract}

Correspondence: Urszula Bazylinska Department of Physical and Quantum Chemistry, Faculty of Chemistry, Wroclaw University of Science and Technology, Wybrzeze Wyspianskiego 27, Wroclaw, 50-370, Poland

Tel +48 7|3202183

Email urszula.bazylinska@pwr.edu.pl

Marcin Nyk

Advanced Materials Engineering and

Modelling Group, Faculty of Chemistry,

Wroclaw University of Science and

Technology, Wroclaw, 50-370, Poland

Tel +48 713202316

Email marcin.nyk@pwr.edu.pl
Introduction: Semiconductor nanoplatelets (NPLs) are promising materials for nonlinear optical microscopy since they feature good two-photon absorption (TPA) properties, narrow photoluminescence spectra and high quantum yields of luminescence. Nevertheless, the use of semiconductor NPLs is inevitably connected with concerns about heavy metal ion toxicity and their intrinsically hydrophobic character.

Methods: Our contribution focuses on the design and engineering of coloidal bionanomaterial consisting of two-dimensional highly luminescent CdSe semiconductor NPLs loaded into spherical and homogeneous polymeric nanocarriers (NCs) based on poly(ethylene oxide) and poly(propylene oxide) block co-polymer. The biocompatibility and usefulness of the NPLs-loaded polymeric NCs in two-photon induced bioimaging was demonstrated invitroby cytotoxicity and two-photon microscopic studies using eukaryotic (normal fibroblasts and cancer ovarian) cells.

Results: The encapsulated NPLs maintain their intensive and spectrally narrow photoluminescence, as well as preserve good TPA properties, while the surrounding polymer shell imparts hydrophilic character and non-toxicity towards eukaryotic cells. Specifically, TPA cross-sections of the colloidal NCs loaded with NPLs show large values reaching up to $2.0 \times$ $10^{8} \mathrm{GM}$, with simultaneously two-photon brightness reaching $2.2 \times 10^{7} \mathrm{GM}$ at $870 \mathrm{~nm}$. MTT proliferation assay performed on cell lines treated with encapsulated NPLs revealed at least $70 \%$ viability of normal human gingival fibroblast (HGF) and cancer ovarian (MDAH-2774) cells, while the results of multiphoton imaging of murine (L-929) fibroblasts suggest that the encapsulated NPLs are capable of labelling the target cells enabling their visualization.

Conclusion: As a result, we obtained water dispersible and temporally stable hydrophilic NPLs-loaded NCs that offer excellent, both one- and two-photon excited fluorescence preserving optical properties of the raw hydrophobic and colloidal NPLs. The biological responses upon eukaryotic cells indicate that the encapsulation process protects cells from the toxic influence of cadmium simultaneously preserving the unique multiphoton properties of the active cargo which opens a promising perspective for its application in multiphoton cancer bioimaging excited at the "optical transmission window" of biological tissues in near-infrared range.

Keywords: semiconductor biomaterials, two-photon luminescence, nanodiagnostic agents, nonlinear optical properties, fibroblasts, cancer ovarian cells, laser diagnostics

\section{Introduction}

Since the seminal report on semiconductor nanoplatelets (NPLs) in $2008,{ }^{1}$ this class of nanomaterials has garnered much attention thanks to their unique optical properties, normally challenging or impossible to achieve in $0 \mathrm{D}$ or 1D nanostructures. 
NPLs are II-VI semiconductor materials (typically made of CdSe, CdS and CdTe), fabricated into small nanostructures whose thickness, as low as several monolayers (ML), can be precisely controlled via synthetic inputs. ${ }^{2}$ NPLs feature high lateral dimensions, and owing to the twodimensional shape, the quantum confinement is dominant along one dimension. For this very reason, NPLs are bracketed together in the group of $2 \mathrm{D}$ materials. High spatial anisotropy translates into a set of hallmark properties of NPLs, ie, characteristically narrow photoluminescence (PL) spectra, high quantum yields (QYs) of luminescence, phenomenally short emission lifetimes, ${ }^{3}$ and as we have recently showed, it can also effect in high exciton binding energies, as shown for CdSe NPLs. ${ }^{4}$ The latter property, assisted by low Auger recombination rates may lead to possible applications in lasing ${ }^{5}$ and in novel photonic devices. ${ }^{6}$ Additionally, quantum yields largely exceed those of 0D quantum dots (QDs), being as high as $45 \% .{ }^{1,7}$ In the field of nonlinear optics, NPLs have also been shown to display high two-photon absorption (TPA) cross-sections, ${ }^{7}$ allowing one to use lower excitation energies in the range of biological transmission window $(650-1000 \mathrm{~nm})$, eg, for nonlinear optical (NLO) microscopy. ${ }^{8}$ An added advantage of the two-photon excitation in near-infrared range is that long-wavelength excitation is much less affected by tissue scattering as well as is less self-absorbed by the biological matter, which in turn minimizes the unwanted autofluorescence background signal. These factors depict why NPLs emerge as promising candidates for photodynamic two-photon cancer therapy ${ }^{9}$ and - owing to the narrow emission peaks - as stains for two-photon bioimaging.

However, despite the attractive linear and NLO properties, bio-applications of NPLs are largely limited due to two factors: the toxicity of cadmium ${ }^{10}$ and their intrinsic hydrophobicity. The latter factor is the reason behind the aggregation of NPLs in biological liquids. The improvement of biocompatibility and colloidal stability of semiconductor nanomaterials has been already approached via numerous functionalization techniques, such as exchange or addition of shorter ligand to the nanoparticle surface (mainly thiols ${ }^{11}$ ), deposition of polymer coating ${ }^{12}$ or encapsulation in amphiphilic polymers, ${ }^{13}$ and encapsulation in micelles, ${ }^{14}$ oil-core nanocarriers, ${ }^{15}$ micro-, and nanoparticles. ${ }^{16}$ While the above techniques generally proved effective in the case of semiconductor nanoparticles, to our best knowledge, colloidal semiconductor NPLs have been successfully transported to the water environment only by ligand exchange method. ${ }^{17,18}$ Nevertheless, the ligand exchange was found to significantly deteriorate the colloidal stability of NPLs as thiol-based ligands tend to detach from NPLs surface upon prolonged storage. Liberated ligands form disulfides, which consequently results in aggregation of NPLs. Wang et al. ${ }^{19}$ abandoned from using thiols in favor of cysteine to perform hydrophilization of CdSe NPLs surface, however as they did not attach any PL spectrum of the resulting material - we followed their ligand exchange protocol, obtaining material with almost four times wider emission spectrum, red-shifted in comparison to raw NPLs (see Figure S1, Supplementary Material). Accordingly, the preparation of reliable solubilization protocols that would enable the transfer of NPLs to water-based media is still an unresolved issue that cries out for a solution.

The present contribution describes thus an efficient approach for the transfer of CdSe NPLs to water-based systems that not only offers high colloidal stability but also allows one to preserve the unique optical properties of the semiconductor cargo. It rests on the encapsulation of the colloidal CdSe NPLs in polymeric nanocarriers (NCs) made of cell-compatible nonionic poly(ethylene oxide) (PEO) and poly(propylene oxide) (PPO) triblock block co-polymers (PEO-PPO-PEO) Pluronic P123 (Scheme 1). Hydrophilic polymeric NCs serve as a barrier between encapsulated NPLs and the biological environment, thus mitigating the exposure to $\mathrm{Cd}^{2+}$ ions, which was reported to be the main source of toxicity in cadmium-based NPs. ${ }^{10}$ An added advantage of the use of polymeric NCs is that their resultant size is much bigger relative to NPLs themselves. This factor may have important implications for cancer diagnosis and treatment, because large NCs would not enter healthy tissues, but are more likely to diffuse into highly permeable tumour cells. ${ }^{20}$ Thus, in our invitrostudies, two human cell lines have been selected, ie, human gingival fibroblasts (HGF) and human ovarian cancer cell line (MDAH), to check both, cytotoxicity and potential biocompatibility of the encapsulated NPLs. The usefulness of NPLsloaded NCs for two-photon bioimaging was demonstrated by two-photon microscopic measurements using murine fibroblasts (L-929 cell line). Collected set of results shows that the NPLs-loaded NCs impart biocompatibility to the active cargo. It is not rare that surface functionalization may render detrimental changes in material optical properties, as was the case with ligand exchange with above-mentioned thiols. However, we demonstrate an encapsulation method which actually preserves the optical properties of CdSe NPLs. The obtained NPLs-loaded NCs maintain their narrow emission peaks which provide a higher advantage of the material as a potential bioimaging agent and - if applied along with 


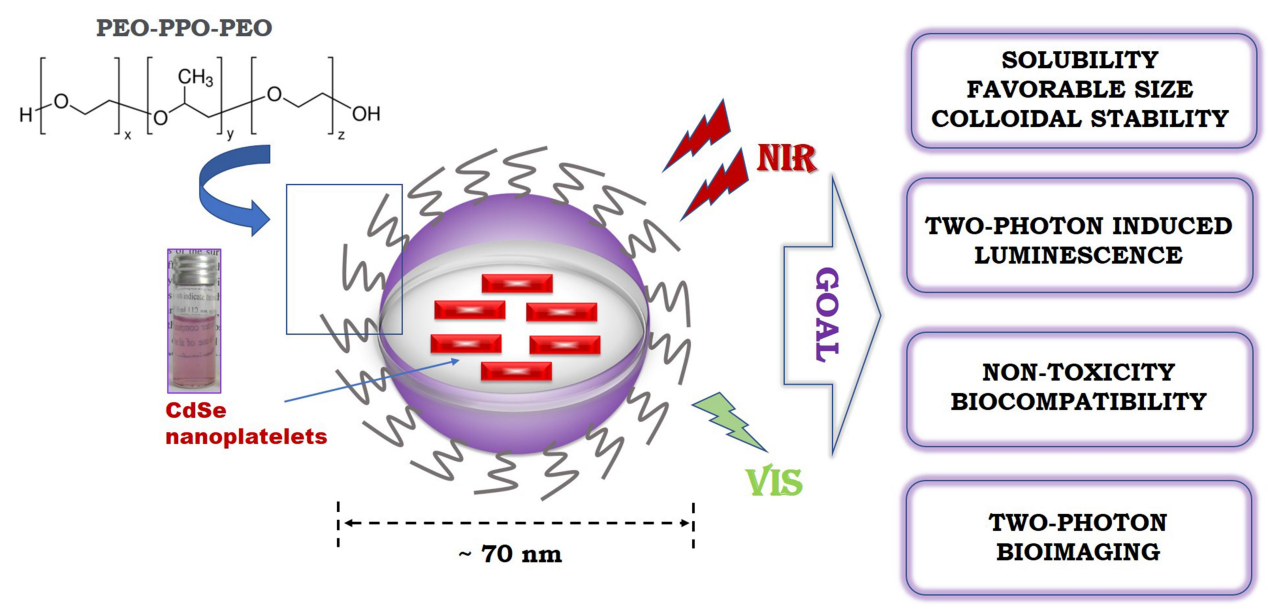

Scheme I The general idea of the performed studies.

NPLs of emission peaks at different wavelengths - enable simultaneous imaging of several phenomena or cell types. However, first and foremost, they display impressive twophoton brightness, to our knowledge never achieved for encapsulated low-dimensional semiconductor nanomaterials, ${ }^{21}$ making them excellent candidates for two-photon bioimaging stains. In brief, conscious of the remarkable optical properties of two-dimensional CdSe NPLs ideal for this application, yet exceeding those of $0 \mathrm{D}$ and $1 \mathrm{D}$ semiconductor nanomaterials, we added a missing property, indispensable for bioimaging ie, biocompatibility - by encapsulating NPLs into polymeric NCs.

\section{Experimental Section Materials}

Sodium myristate $(\geq 99 \%)$, cadmium nitrate tetrahydrate (99.997\%), selenium powder $(99.99 \%)$, cadmium acetate hydrate ( $\geq 99.99 \%)$ and 1-octadecene (technical grade, 90\%) were purchased from Sigma Aldrich. Chloroform ( $\geq 99.9 \%$ ) was purchased from P.P.H. "STANLAB" (Poland). 1-butanol ( $\geq 99.999 \%$ ) was purchased from Avantor Chemicals (Poland). Poly(ethylene oxide) (PEO) and poly(propylene oxide) (PPO) block co-polymer (PEO-PPO-PEO) - Pluronic P-123 was purchased from Sigma Aldrich.

\section{Cd Precursor Synthesis}

The cadmium precursor was prepared at room temperature by adapting the method described by Tessier et al. ${ }^{22}$ Briefly, $3.13 \mathrm{~g}$ of sodium myristate was dissolved in $250 \mathrm{~mL}$ of methanol with strong stirring. In another flask, $1.23 \mathrm{~g}$ of cadmium nitrate was dissolved in $40 \mathrm{~mL}$ of methanol. Two resulting solutions were mixed and a white precipitate appeared after a while. The precipitate was filtered out and washed with methanol using a Buchner vacuum kit and dried overnight.

\section{CdSe NPLs Synthesis}

To synthesize colloidal 5.5 ML CdSe nanoplatelets, we followed a protocol described previously by Ithurria et $\mathrm{al}^{1}$ with some modifications described in our previous paper. ${ }^{4}$ Briefly, $12 \mathrm{mg}$ of selenium powder, $85 \mathrm{mg}$ of prepared cadmium myristate, and $15 \mathrm{~mL}$ of octadecene were mixed in a threeneck flask. The flask was purged with dry nitrogen at $10 \mathrm{~min}$ intervals and then degassed under vacuum. The mixture was heated to $240^{\circ} \mathrm{C}$ under nitrogen in a Schlenk line. When the temperature reached $195^{\circ} \mathrm{C}, 60 \mathrm{mg}$ of cadmium acetate was quickly introduced into the flask. The mixture was maintained at $240^{\circ} \mathrm{C}$ for $10 \mathrm{~min}$. After cooling to ambient temperature, the mixture was centrifuged for $10 \mathrm{~min}$ (3000 rpm) with 1-butanol. The precipitate was redispersed and sonicated in chloroform. The centrifugation with 1-butanol was repeated once again at the same conditions and the final product was redispersed in chloroform.

\section{NPLs-Loaded NCs Preparation}

NPLs-loaded NCs were prepared via solvent-evaporation method, developed previously by our group. ${ }^{15}$ Briefly, 3\% w/w Pluronic P-123 dichloromethane solution was added to $1.17 \mathrm{mg} / \mathrm{mL}$ chloroform solution of the synthesized $\mathrm{CdSe}$ NPLs in a round-bottomed flask. We prepared 6 different polymer-nanoparticle combinations with CdSe NPLs solution to Pluronic solution to water volume ratios presented in Table S1, (Supplementary Material). The excess of solvent 
was evaporated using a rotary evaporator (Hei-VAP Value Digital, Heidolph Instruments, Schwabach, Germany) with a rotation speed of $150 \mathrm{rpm}$. Subsequently, the flask was placed in a $45^{\circ} \mathrm{C}$ water bath and $5 \mathrm{~mL}$ of distilled water was added to the flask. The mixture was agitated using a magnetic stirrer, the remaining organic solvents were evaporated, and the product was cooled down overnight.

\section{Physicochemical Characterization Methods}

\section{Morphology Evaluation by Transmission Electron Microscopy}

The morphology and shape of the prepared CdSe NPLs and NPLs-loaded NCs were examined with transmission electron microscopy (TEM) using a Hitachi H-800 electron microscope. The specimens were prepared by placing a few drops of diluted NPLs or NPLs-loaded NCs on $\mathrm{Cu}$ $\mathrm{Ni}$ grids and left to dry for $1 \mathrm{~h}$ at room temperature before observation. EDX (energy-dispersive X-ray spectroscopy) analysis was conducted on a JEOL JSM-6610LVnx scanning electron microscope with attached EDX probe.

\section{One-Photon and Two-Photon Optical Characteristics}

Firstly, the prepared colloidal nanomaterials were subjected to linear optical characterization. The UV-VIS absorption spectra were measured using a JASCO V-670 spectrophotometer. The photoluminescence measurements were performed with a Hitachi F-4500 spectrofluorometer, under $350 \mathrm{~nm}$ excitation. Time-resolved photoluminescence measurements were performed using a system consisting of a picosecond $375 \mathrm{~nm}$ laser diode (BDL-375$\mathrm{SMC}$ ) as an excitation source and a custom-made Time Correlated Single Photon Counting (TCSPC) Becker \& Hickl system, built on a TCSPC Module (SPC-130-EM) and a hybrid PMT detector (HPM-100-06) with a detector control card (DCC 100) set onto a Princeton Instruments spectrograph (ActonSpectraPro-2300i). Biexponential decay model was used for the photoluminescence lifetimes calculation, and the least-squares fitting was performed with the use of the dedicated Becker \& Hickl SPCImage software.

The spectrally resolved TPA properties of the synthesized CdSe NPLs and NPLs-loaded NCs were studied following the procedure described by Makarov et $\mathrm{al}^{23}$ using two-photon excited emission (TPEE) method based on comparison between the emission intensities of the investigated materials with the corresponding signals of
Rhodamine $\mathrm{B}(\mathrm{RhB})$ dye. The $\sigma_{2}$ values were determined with the accuracy of $\pm 15-20 \%$. As an excitation source was employed a laser system consisting of a Quantronix Integra-C Ti:sapphire regenerative amplifier which generates $130 \mathrm{fs}, 800 \mathrm{~nm}$ pulses with $1 \mathrm{~mJ}$ energy per pulse and at a $1 \mathrm{kHz}$ repetition rate pumping a Quantronix Palitra-FS optical parametric amplifier, capable of output wavelength tuning between 530 and $2000 \mathrm{~nm}$.

\section{Photochemical Photostability Verification}

Photochemical photostability of NPLs-loaded polymeric NCs was tested by detecting PL intensity with respect to $\mathrm{pH}$ and temperature under the excitation wavelength $\left(\lambda_{\text {exc. }}\right)$ of $350 \mathrm{~nm}$ using FluoroMax-4 Spectrofluorimeter (Horiba Scientific). The NPLs-loaded NCs solution was incubated in ThermoMixer $\mathrm{C}$ (Eppendorf) at $25^{\circ} \mathrm{C}, 30^{\circ} \mathrm{C}, 33^{\circ} \mathrm{C}, 37^{\circ}$ $\mathrm{C}$ and $40^{\circ} \mathrm{C}$ or their $\mathrm{pH}$ was adjusted between 6 and 9.5 by using $\mathrm{HCl}$ and $\mathrm{NaOH}$. Each measurement was repeated a few times.

\section{Biological Experiments in vitro Cell Viability Assay}

The biocompatibility of the encapsulated NPLs was tested in vitro using normal human gingival fibroblasts from primary culture (HGF, normal cell line) and human ovarian endometrioid adenocarcinoma cell line, sensitive to cisplatin (MDAH-2774). Primary HGFs were isolated from a fragment of gingival tissue (1-2 mm) obtained from healthy patients, according to the procedure previously described by Saczko et al. ${ }^{24}$ The isolation procedure and cultivation experiments were conducted in agreement with the requirements of the Bioethics Commission of Wroclaw Medical University. The use of the HGF cell lines had ethical and institutional review board (Bioethical Committee of Wroclaw Medical University, Poland) approval (approval, no: KB-8/2010). The ovarian cancer cell line was purchased from ATCC $^{\circledR}$ (Manassas, VA, USA), cultured and prepared according to the conditions described previously by our group. ${ }^{25}$ The in vitro cytotoxicity evaluation of the studied NCs loaded with NPLs in four different dilutions (1:50, 1:100, 1:200, 1:500) was performed using an MTT reduction assay, after $24 \mathrm{~h}$ of cell treatment. The measurements were performed using the GloMax ${ }^{\circledR}$ Discover multimode microplate reader (Promega). The cell viability in each group was expressed as a percentage of the value obtained for control (untreated) cells (average of three experiments). 


\section{Two-Photon Bioimaging Studies}

The two-photon bioimaging studies were performed on murine fibroblasts (L-929 cell line, ATCC $® C C L-1^{\mathrm{TM}}$ ) in the logarithmic growth phase, which were seeded onto sterile round glass coverslips and placed in a 24-well plate at a density of $0.5 \times 10^{5}$ cells/well in Iscove's Modified Dulbecco's Medium (GIBCO) with $10 \%$ fetal bovine serum (GIBCO) and antibiotics (100 U/ $\mathrm{mL}$ penicillin, $0.1 \mathrm{mg} / \mathrm{mL}$ streptomycin, GIBCO). After 24 $\mathrm{h}$ cells were treated with encapsulated CdSe NPLs (loaded NCs) or empty NCs diluted $25 \mathrm{x}$ from a $1 \mathrm{mg} / \mathrm{mL}$ stock solution. Next, cells were washed three times with PBS to remove free NCs and fixed with $4 \%$ formaldehyde. For imaging, cells and free NCs (loaded and empty) were mounted in Prolong Gold anti-fade solution. The bioimaging was performed on a Leica SP8 MP microscope equipped with femtosecondpulsed infrared (IR) laser Chameleon Vision II (Coherent) and spectral PMT detectors using a $63 \mathrm{x}$ oil objective (HC PL APO CS2 63x/1.4 OIL). To visualize NPLs two-photon excitation and emission scans were performed in order to establish optimal imaging conditions (the results are given in the Supplementary Material). For the excitation scan, NPLs were imaged at various excitation wavelengths between 680 and $1080 \mathrm{~nm}$ with $20 \mathrm{~nm}$ step and 500-600 nm emission range. Since the IR laser power varies depending on the wavelength from $0.78 \mathrm{~W}$ at $680 \mathrm{~nm}$ to $0.34 \mathrm{~W}$ at $1080 \mathrm{~nm}$ with a peak of 3.6 $\mathrm{W}$ at $800 \mathrm{~nm}$ fluorescence intensities of NPLs were normalized to allow for comparison of the excitation efficiency (Figure S5, Supplementary Material). For the emission scan, the $870 \mathrm{~nm}$ excitation wavelength was chosen and NPL emission spectrum was recorded at 19 steps (each with $20 \mathrm{~nm}$ range) covering the total 400-780 nm range (Figure S6, Supplementary Material). Cells incubated with NPLs were imaged using a reflection mode with a $638 \mathrm{~nm}$ laser line to visualize cell bodies while NPLs were finally visualized at $870 \mathrm{~nm}$ excitation and $525-575 \mathrm{~nm}$ emission range. $Z$ stacks of 8-bit images encompassing a distance of $16 \mu \mathrm{m}$ were acquired to cover the full volume of adherent L-929 cells. At least five randomly chosen areas were imaged for each condition. The obtained images were analyzed using the ImageJ software (NIH).

\section{Results and Discussion Structural and Optical Characterization of Free and Encapsulated CdSe NPLs}

The morphology of the raw synthesized 5.5 ML CdSe NPLs is presented using TEM technique in Figure 1A together with their size distribution histograms in Figure 1B and C. Both dimensions exhibit Gauss distribution with an average length of $25.5 \pm 2.3 \mathrm{~nm}$ and a width of $9.3 \pm 1.3 \mathrm{~nm}$. Nanoparticles of this size may not be suitable as diagnostic agents for biological application due to the renal clearance risk. ${ }^{26}$ As shown in Figure 2, the CdSe NPLs, after loading them into polymer nanocarriers, show a size of approximately $80 \mathrm{~nm}$, which will perfectly fit into biological requests including optical based, non-contact diagnostics due to their longer circulation time in the bloodstream. ${ }^{27}$

Figure 2 shows TEM-imaged morphology of these CdSe NPLs encapsulated in Pluronic P-123 NCs for the sample 5 contained the most homogeneous, monodisperse and spherical NCs (Figure 2) as well as the best luminescent properties. Figure S2, Supplementary Material presents energy-dispersive X-ray (EDX) spectrum of the encapsulated CdSe NPLs. The characteristic peaks of cadmium and selenium indicate that indeed nanocarriers contain CdSe NPLs. When a larger volume of polymer was used (sample 6) at the same CdSe NPLs concentration, the obtained NCs had less controlled shape and size distribution (Figure S3, Supplementary Material). We do not recommend higher dilutions at the preparation stage (samples 1-4) as the obtained nanosystems display poorer sphericity and homogeneity, losing the PL properties. The challenge of the encapsulation was to enclose the CdSe NPLs in spherical polymeric NCs with a hydrophilic shield, simultaneously preserving their optical properties. Therefore, we performed PL measurements for all six samples (Figure 3A) in order to choose the best composition for further optical and biological characterization. The highest PL intensity was exhibited by sample 5, which may be simply related to the final concentration of the luminescent CdSe NPLs in NCs (the highest among all samples) and the optimal concentration of the polymer. All emission spectra of the encapsulated CdSe NPLs, no matter the composition, display an additional broad band located between $400 \mathrm{~nm}$ and about $530 \mathrm{~nm}$. Such a peak is characteristic for emission of organic backbone of Pluronic P-123 and may be seen as an auxiliary confirmation that the encapsulation process indeed took place.

To study the impact of encapsulation on the optical properties of the 2D semiconductor NPs, we measured the optical density and PL intensity spectra (Figure $3 \mathrm{~B}$ and $\mathrm{C}$ ) as well as PL time-resolved decays (Figure 3D) for both CdSe NPLs and NPLs-loaded NCs. As it has been already reported in numerous works, ${ }^{2-4}$ the absorption spectra of CdSe NPLs contain characteristic multiple peaks. First, two of them are attributed to the transitions from the heavy-hole and lighthole valence bands to the conduction band, respectively, ${ }^{28}$ among which the heavy-hole transition peak at $552 \mathrm{~nm}$ is 

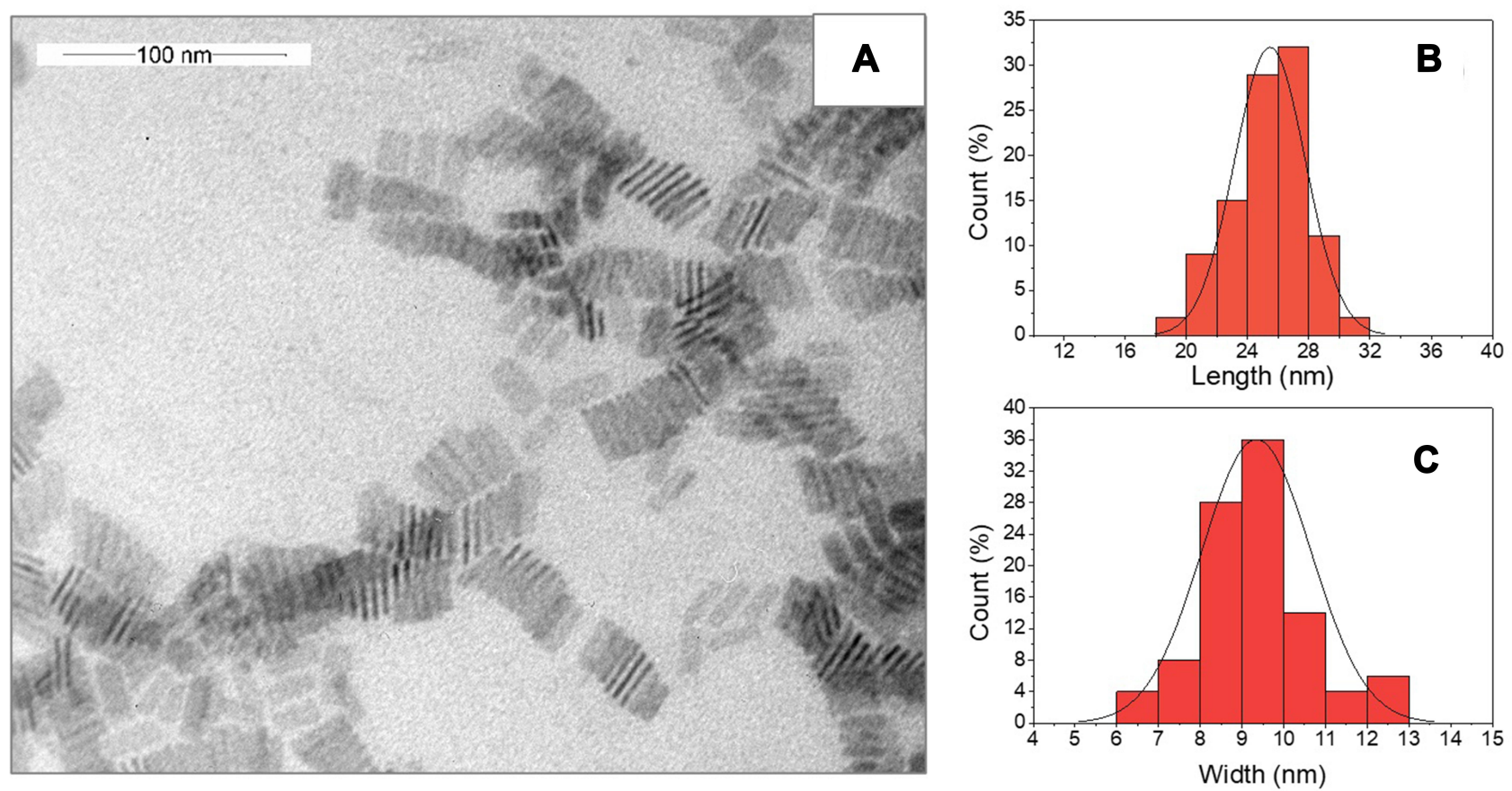

Figure I TEM image of 5.5 ML CdSe NPLs (A) along with their length (B) and width (C) distributions.
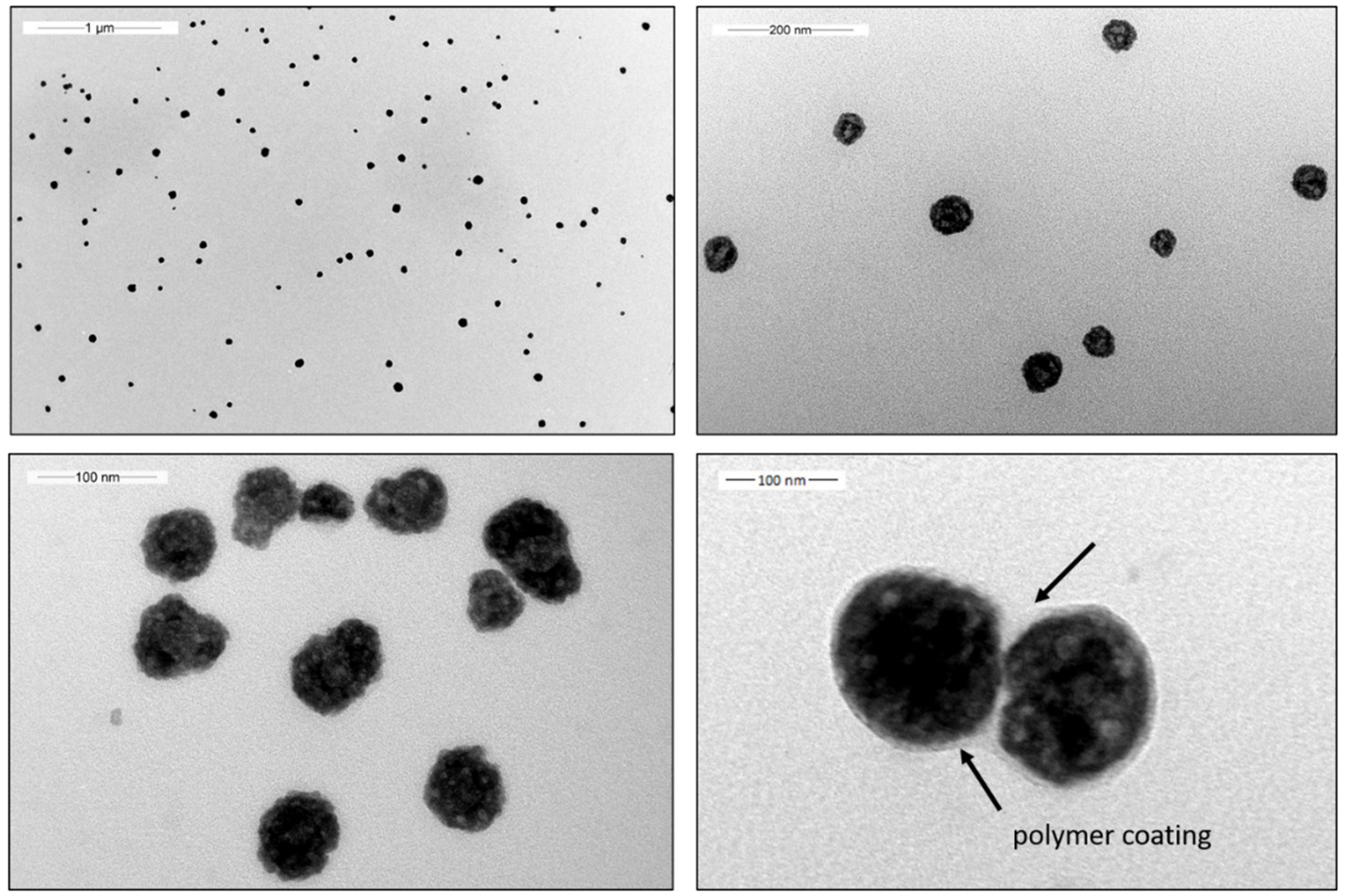

Figure 2 Exemplary TEM images of CdSe NPLs encapsulated in Pluronic NCs. The arrows indicate pale polymeric shell in contrast to dark inorganic load inside. 

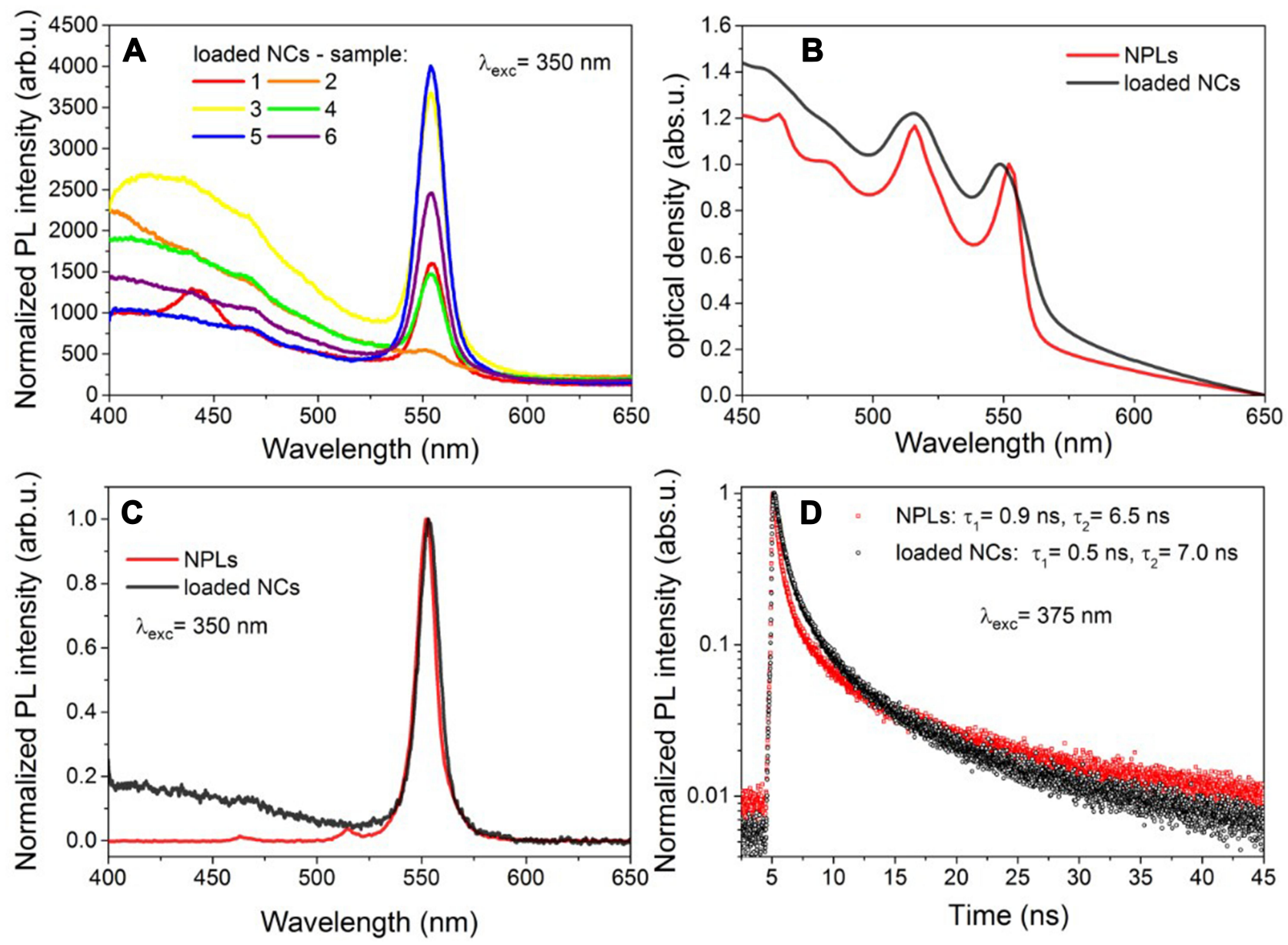

Figure 3 One-photon optical characterization of NPLs and NPLs-loaded NCs: (A) the impact of samples composition (detailed in Table SI, Supplementary Material) on PL intensities (B) absorption spectra, (C) luminescence spectra, (D) fluorescence intensity decays.

particularly sharp. In the emission spectrum of 5.5 ML CdSe NPLs one can observe a narrow and uniform peak at $553 \mathrm{~nm}$ with a full width at half-maximum (FWHM) of $10.9 \mathrm{~nm}$. From the time-resolved PL measurements, by fitting with biexponential decay function, we calculated the decay lifetime values to be ca. $\tau_{1}=0.9 \mathrm{~ns}, \tau_{2}=6.5 \mathrm{~ns}$, which are of the same order of magnitude as those reported previously (at room temperature). ${ }^{3,29,30}$ The most important, and at the same time the most promising result is that neither FWHM, nor the position of the emission peak is significantly changed after the encapsulation process. Indeed, NPLs-loaded NCs exhibit the highest emission intensity at $553 \mathrm{~nm}$ with a FWHM of $12.2 \mathrm{~nm}$. In the optical density spectrum, one can observe a slight smoothing of the sharp slopes and a small blue shift of the absorption envelope, resulting in the maximum absorption for the first excitonic peak at 549 $\mathrm{nm}$; however, all peaks are still clearly visible. Time-resolved PL measurements displayed similar decay lifetime of encapsulated NPLs with values of $\tau_{1}$ and $\tau_{2}$ equal to $0.5 \mathrm{~ns}$ and $7.0 \mathrm{~ns}$, respectively. Additionally, we have verified how the dilution of the chosen sample influences its PL intensity and we have detected a reasonable signal even for a 20 times diluted NPLs-loaded NCs solution.

Encouraged by these promising results, showing that the encapsulation process has only a minor effect on the optical properties of CdSe NPLs, we went a step further and performed spectrally resolved TPA measurements of both NPLs and NPLs-loaded NCs in order to investigate the impact of encapsulation on TPA cross-sections as well as to characterize NPLs-loaded NCs themselves as unitary systems, treating them as if they were a new-type hybrid NPs. Due to the limited coverage of the literature on NLO properties of $2 \mathrm{D}$ semiconductor nanoparticles, ${ }^{7}$ we measured two-photon excited photoluminescence properties of the synthesized NPLs (Figure 4A) and NPLs-loaded NCs (Figure 4B) in a wide range of excitation wavelengths 


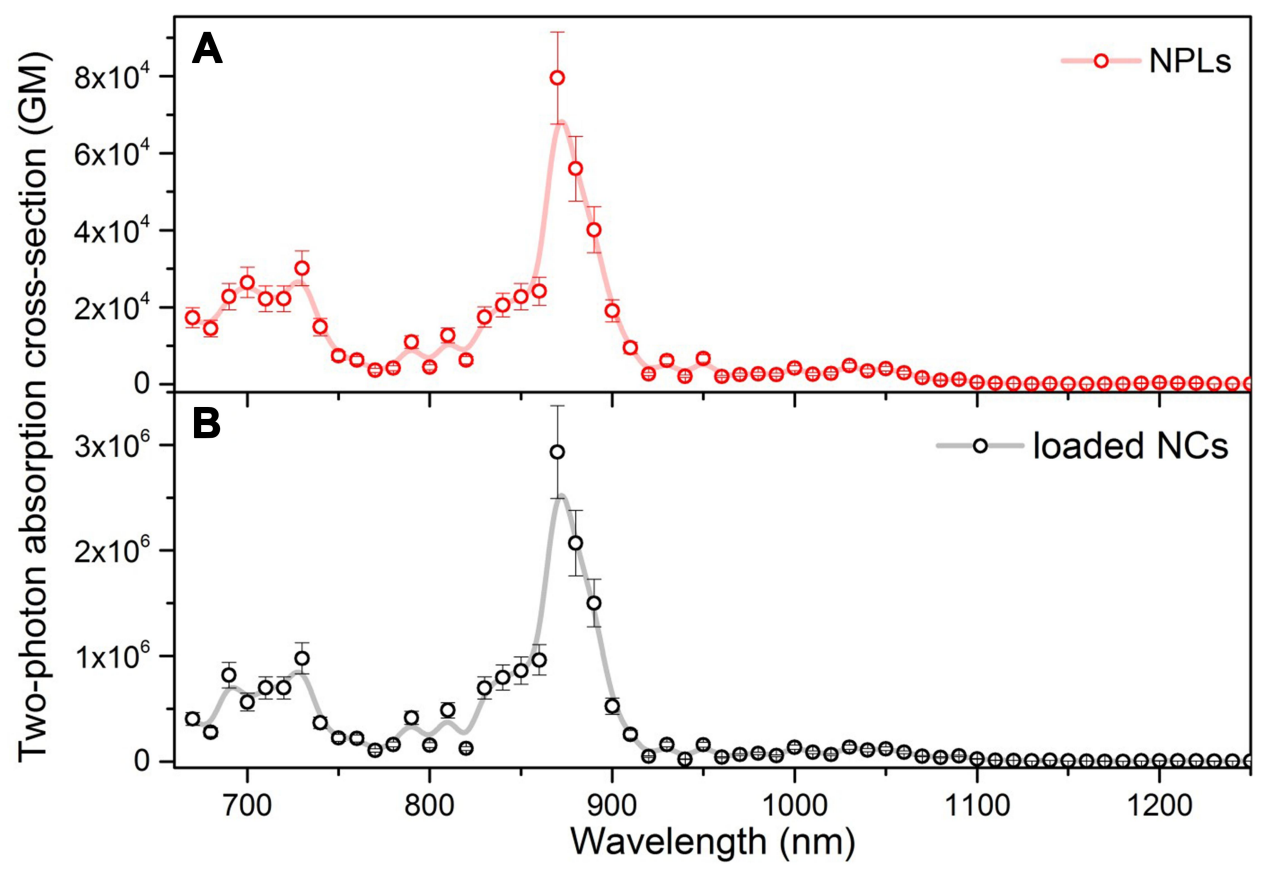

Figure 4 Wavelength dependence of two-photon absorption cross-section of NPLs (A) and NPs-loaded in NCs (B) measured by TPEE technique. The lines led through experimental points are drawn just to guide eyes.

(670-1250 $\mathrm{nm}$ ) in order to complement the missing knowledge in this area.

The representative two-photon induced emission spectra of the NPLs and NPLs-loaded NCs under $870 \mathrm{~nm}$ femtosecond laser excitation are shown in Figure S4, Supplementary Material. We found that the position of the maximum of two-photon induced emission mirrors that obtained under one-photon excitation conditions at $350 \mathrm{~nm}$ for both NPLs and NPLs-loaded NCs (see Figure 3C). Figure $4 \mathrm{~A}$ and $\mathrm{B}$ presents the wavelength dependence of the effective TPA cross-section $\sigma_{2}$ determined using TPEE method for the NPLs and NPLs-loaded NCs. In the case of NPLs-loaded NCs, it was assumed that a single NC contains 2000 NPLs. Collected results show that the maximum TPA cross-section of $8.0 \times 10^{4} \mathrm{GM}$ (Goeppert-Mayer units, where $1 \mathrm{GM}=10^{-50} \mathrm{~cm}^{4} \mathrm{~s}_{\text {photon }}{ }^{-1}$ ) for NPLs of $357 \mathrm{~nm}^{3}$ is found at $870 \mathrm{~nm}$; this is a similar result to that reported by Scott et $\mathrm{al}^{7} 3.43 \times 10^{5} \mathrm{GM}$ for NPLs of $346 \mathrm{~nm}^{3}$. In the case of NPLs-loaded NCs, the TPA cross-section is $2.0 \times 10^{8} \mathrm{GM}$ at the same wavelength. However, a common practice is to perform a normalization procedure of TPA to account for the overall mass (or size) of the nanoobject, so that TPA response of nanoparticles of different sizes could be compared in more quantitative terms. Thus, the particle weight normalized TPA cross-section parameter has been calculated $\left(\sigma_{2} / \mathrm{M}\right.$, where $\mathrm{M}$ is the mass of a mole of a given kind of nanoparticle).

As seen in Table 1 , the $\sigma_{2} / \mathrm{M}$ parameters for loaded NCs and NPLs are of the same order of magnitude. It indicates that the encapsulation has practically no negative impact on TPA crosssection of NPLs. Notably, we have also calculated two-photon action cross-sections $\left(\sigma_{2} \cdot \eta\right)$, because for applications such as two-photon microscopy the most relevant parameter is the effective intensity of two-photon excited luminescence, rather than the intrinsic TPA itself (Table 1). In order to compare the brightness of a NPL in chloroform with the brightness of a NPL enclosed in polymeric NCs and dispersed in water, we additionally calculated

Table I Quantitative Evaluation of Quantum Yield and Two-Photon Absorption Cross-Section of CdSe NPLs and CdSe NPLs-Loaded NCs at $870 \mathrm{~nm}$ Together with Rhodamine B Which Was Used as a Reference

\begin{tabular}{|l|c|c|c|c|c|c|}
\hline & Solvent & $\boldsymbol{\eta}^{\mathbf{a}}[\%]$ & $\boldsymbol{\sigma}_{\mathbf{2}}^{\mathbf{b}} \mathbf{( G M )}$ at $\mathbf{8 7 0} \mathbf{~ n m}$ & $\left.\boldsymbol{\sigma}_{\mathbf{2}} / \mathbf{M}^{\mathbf{c}} \mathbf{( G M} \cdot \mathbf{m o l} / \mathbf{g}\right)$ & $\boldsymbol{\sigma}_{\mathbf{2}} \cdot \boldsymbol{\eta}^{\mathbf{d}}(\mathbf{G M})$ & $\left(\boldsymbol{\sigma}_{\mathbf{2}} / \mathbf{M}\right) \cdot \boldsymbol{\eta}^{\mathbf{e}}(\mathbf{G M} \cdot \mathbf{m o l} / \mathbf{g})$ \\
\hline Rhodamine B & $\mathrm{CH}_{3} \mathrm{OH}$ & $45[2 \mathrm{I}]$ & $74[2 \mathrm{I}]$ & 0.154 & 33.3 & $6.9 \cdot 10^{-2}$ \\
CdSe NPLs & $\mathrm{CHCl}_{3}$ & 45 & $8.0 \cdot 10^{4}$ & $6.4 \cdot 10^{-2}$ & $3.6 \cdot 10^{4}$ & $2.9 \cdot 10^{-2}$ \\
CdSe NPLs-loaded NCs & $\mathrm{H}_{2} \mathrm{O}$ & $\mathrm{II}$ & $2.0 \cdot 10^{8}$ & $7.8 \cdot 10^{-2}$ & $2.2 \cdot 10^{7}$ & $1 \cdot 10^{-3}$ \\
\hline
\end{tabular}

Notes: ${ }^{a}$ Quantum yield, ${ }^{b}$ two-photon absorption cross-section, ${ }^{c}$ particle weight normalized two-photon absorption cross-section, ${ }^{d}$ two-photon action cross-section, eparticle weight normalized two-photon action cross-section. 
particle weight normalized two-photon brightness $\left(\sigma_{2} / \mathrm{M}\right) \cdot \eta$. It turns out that although the QY of luminescence of NPLs-loaded NCs is ca. four times lower than that for NPLs (meaning that encapsulation introduces some non-radiative decay pathways, likely due to the formation of sub-aggregates of NPLs within $\mathrm{NCs})$, the final $\left(\sigma_{2} / \mathrm{M}\right) \cdot \eta$ value of NPLs-loaded NCs is comparable to that of pristine NPLs.

In order to ensure that the NPLs-loaded NCs are stable in actual human organism conditions, we have studied their photochemical photostability by detecting their PL intensity at different $\mathrm{pH}$ and temperature values. Figure 5 presents that the emission intensity of encapsulated CdSe NPLs does not change significantly in a range of physiologically relevant $\mathrm{pH}(6-9.5)$, and temperature $\left(25-40^{\circ} \mathrm{C}\right)$ conditions. We observed no more than $15 \%$ of the change in maximum fluorescence

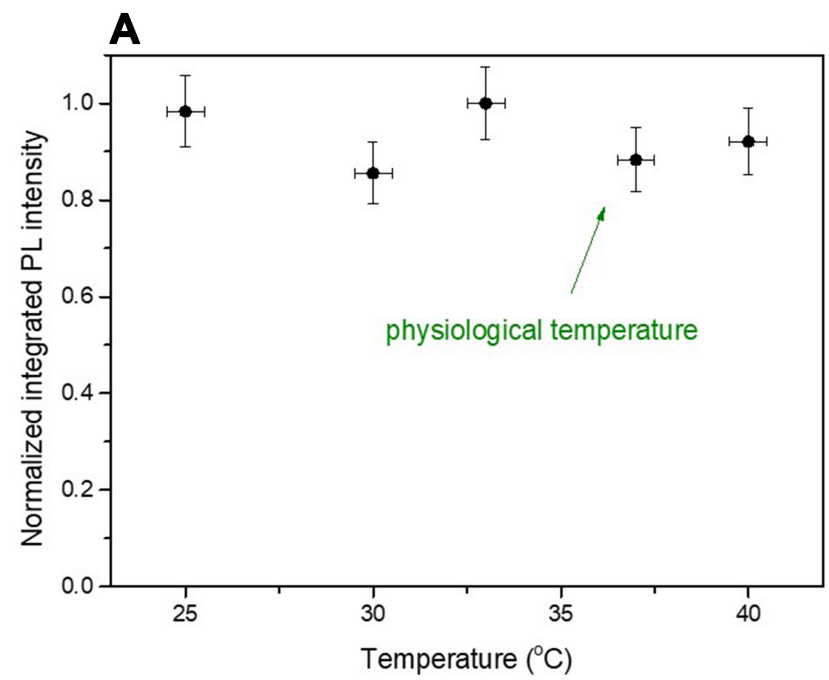

B

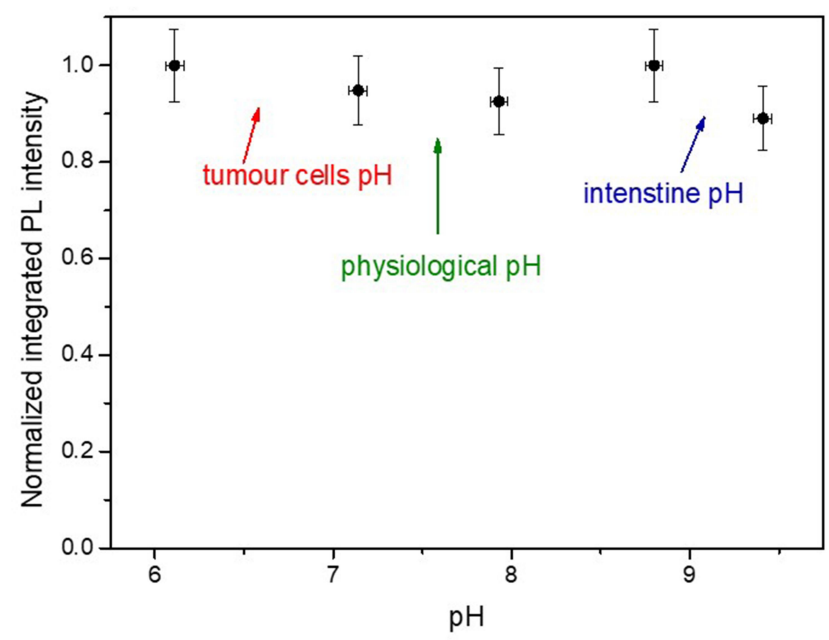

Figure 5 Stability of NPLs-loaded NCs at different $(\mathbf{A})$ temperature and $(\mathbf{B}) \mathrm{pH}$ conditions. intensity throughout these experimental ranges. The demonstrated photostability is a strong advantage for bioimaging applications with normal human body temperature within $36.5^{\circ} \mathrm{C}$ and $37.5^{\circ} \mathrm{C}$, blood $\mathrm{pH} \sim 7.4$ and some tumour cells $\mathrm{pH} \sim 6.5$.
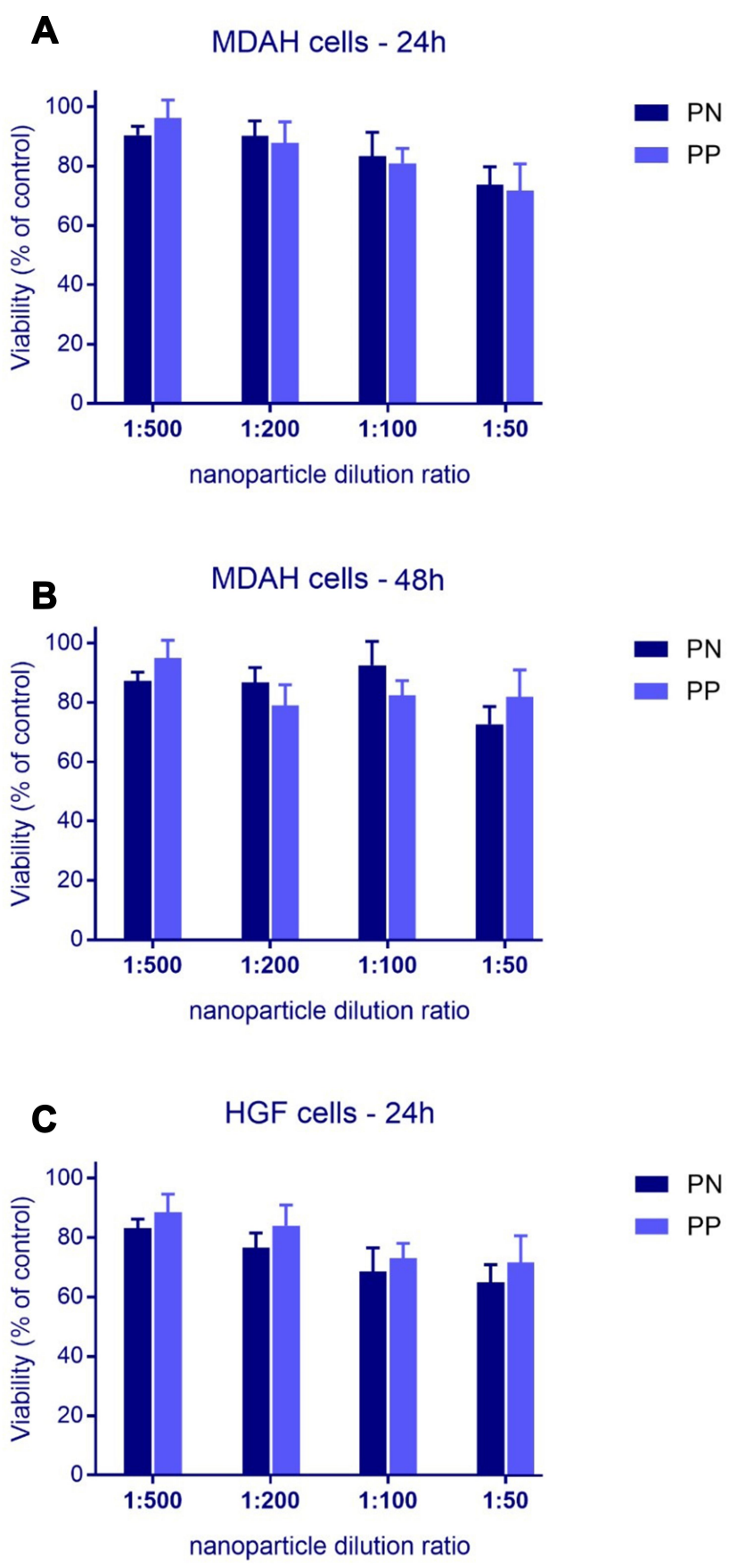

Figure 6 Cytotoxicity evaluation in human ovarian endometrioid adenocarcinoma cell line, MDAH-2774 (A and B) and normal human gingival fibroblasts, HGF (C) after treatment with encapsulated CdSe NPLs (PN) and empty NCs (PP). Results are expressed as mean \pm SD. 


\section{In vitro Biological Response of Encapsulated CdSe NPLs}

If NPL-loaded NCs are considered as NLO stains for twophoton microscopic observations of biological matter, of utmost importance is the evaluation of their potential toxicity against potential target cells. Thus, first, we have assessed the appropriate "dark" cytotoxicity (Figure 6) of the NCs loaded with CdSe NPLs (PN) and empty ones (PP) in in vitro cultures of normal human gingival fibroblasts (HGF) and human ovarian endometrioid adenocarcinoma cell line, (MDAH-2774), with the use of MTT viability assay. As it has been shown previously, fluorescent semiconductor cadmium-based nanomaterials can directly cause cytotoxic damage not only to cancer cells, but also to normal tissues. ${ }^{31,32}$ Thus, encapsulation techniques may reduce deleterious toxic effects and increase the biocompatibility of the luminescent cargo. Our in vitro cytotoxicity experiments indicate that both, the empty and loaded NCs show no toxic effect in MDAH-2774 cells (cell viability above 70\%), even at higher concentrations (dilution 1:50) after prolonged incubation time ( $48 \mathrm{~h}$ ). Furthermore, no toxic effect toward the normal cell line (HGF) additionally proved the preserving effect of the NCs polymer shell and the potential of the encapsulated NPLs for in vivo applications.
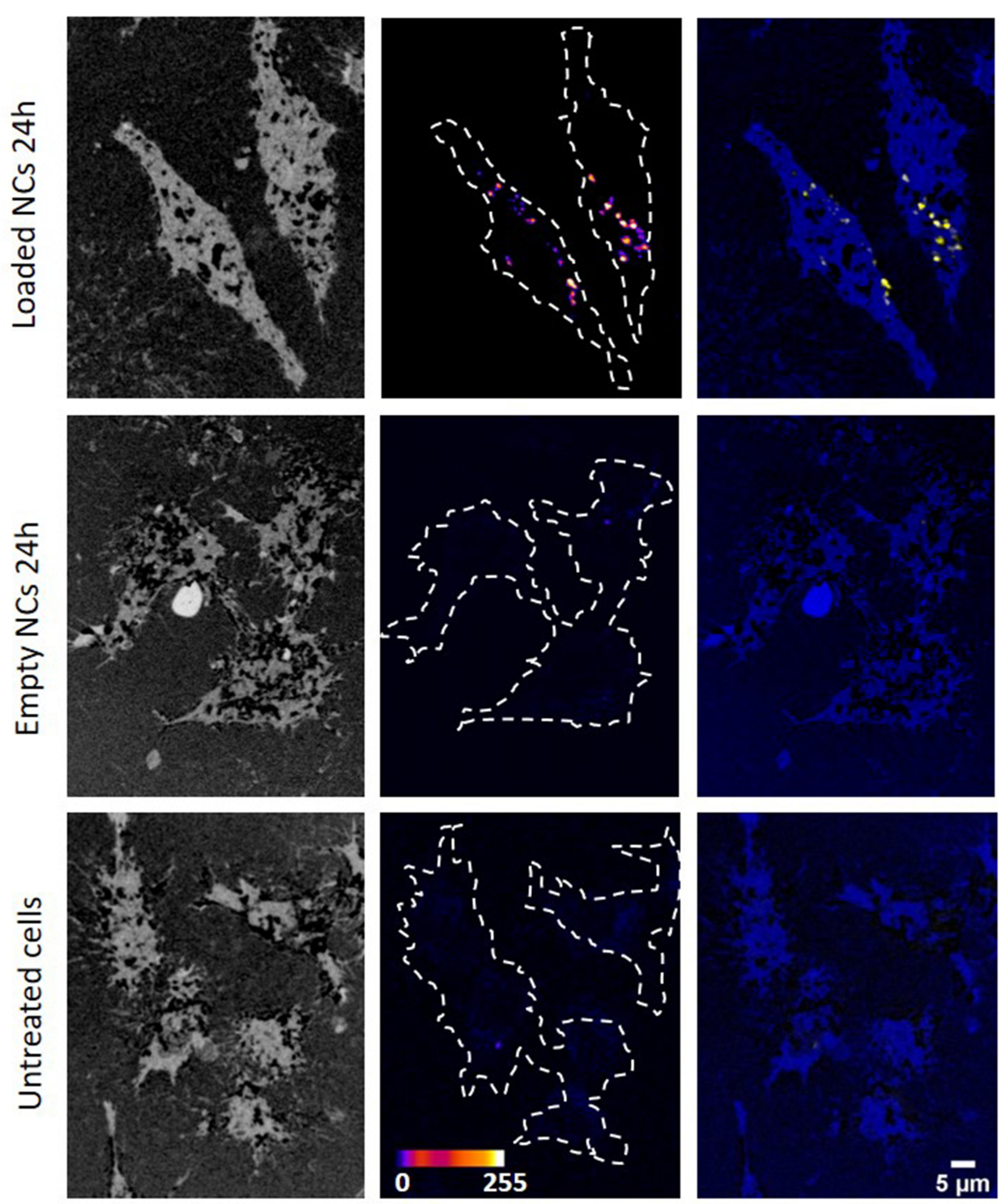

Figure 7 Two-photon microscopy of fibroblasts incubated with NPL-loaded NCs. Cell outlines (white dashed lines) were drawn based on the reflection channel (shown in grey scale in left panel or blue in overlaid image). NPL channel presented in fire look-up table (middle panel) and in yellow in overlaid image. Representative single optical sections of cell bodies are shown. 
The intrinsic fluorescence of the encapsulated NPLs allowed us to employ two-photon microscopy to study their potential for diagnostic two-photon bioimaging. Microscopic analysis of fibroblast cell cultures incubated with NPLloaded NCs for $24 \mathrm{~h}$ revealed that cells were able to survive in the presence of high doses of NCs, confirming the results of cytotoxicity experiments performed on human cell lines. Notably, we observed a substantial decoration of the plasma membrane with NCs with concomitant partial internalization of NCs into the cytoplasm of fibroblasts (Figure 7) despite the fact that no additional stimulation was employed during the chosen incubation time. Nevertheless, to enhance the cellular penetration of the encapsulated NPLs for further in vivo studies, increasing the incubation time or additional stimulation methods such as electroporation may be beneficial. ${ }^{25}$

\section{Conclusions}

An important objective of this work was to characterize NLO properties of encapsulated CdSe NPLs in a polymeric nanocontainer based on Pluronic P123 as an emerging new class of colloidal hybrid materials, for potential applications in nanophotonics and biophotonics. Studied semiconductor NPLs are promising materials for NLO microscopy (showing advantages over $0 \mathrm{D}$ and 1D materials) since they feature high twophoton absorption cross section, narrow photoluminescence spectra and high quantum yields of luminescence. Moreover, their extremely narrow luminescence peaks increase merit of the final functional hybrid nanomaterial as a future bioimaging agent and opens the possibility for imaging several processes/ cell types simultaneously when using NPLs with luminescence maxima at different wavelengths. While the literature contains many attempts of evaluation of NLO properties of various nanostructures, much of the reported work does not provide sufficiently scrupulous data to allow for comparisons of the relative merit factors of such structures and their optimization. Therefore, in the present work, we called for the developing of host-guest systems, in which the host is composed of hydrophilic polymeric nanocarrier and as guests CdSe NPLs are employed. As a result, we obtained waterdispersible and temporally stable hydrophilic CdSe NPLsloaded NCs that offer excellent, both one- and two-photon excited fluorescence preserving optical properties of the raw hydrophobic and colloidal NPLs. The MTT assay results indicate that the encapsulation process protects cells from the toxic influence of cadmium. Moreover, multiphoton microscopy shows the CdSe NPLs-loaded NCs attached or internalized to target cells enabling their visualization. All of these results together make this new hybrid nanomaterial a biocompatible - from both bioliquids affinity and cytotoxicity points of view - and efficient two-photon fluorescence agent which opens a promising perspective for its application in multiphoton cancer bioimaging.

\section{Abbreviations}

NPLs, nanoplatelets; TPA, two-photon absorption; NCs, nanocarriers; HGF, human gingival fibroblast; ML, monolayers; PL, photoluminescence; QY, quantum yield; QDs, quantum dots; NLO, nonlinear optical; PEO, poly(ethylene oxide); PPO, poly(propylene oxide); MDAH, human ovarian cancer cell line; TEM, transmission electron microscopy; TCSPC, Time Correlated Single Photon Counting; TPEE, two-photon excited emission; RhB, Rhodamine B; IR, infrared; FWHM, full width at half-maximum; PN, NCs loaded with CdSe NPLs; PP, empty NCs.

\section{Acknowledgment}

We acknowledge Dr Andrzej Żak for TEM imaging experiment.

\section{Supplementary Material}

The following Supplementary Material is available free of charge at the Dove Press website: samples composition, TEM image of another sample, exemplary TPEE spectra, two-photon microscopy analyses.

\section{Funding}

Research funded by National Science Centre, Poland under Grant no. 2018/29/B/ST4/02172 and by Wroclaw University of Science and Technology.

\section{Disclosure}

The authors declare no competing financial or other interests.

\section{References}

1. Ithurria S, Dubertret B. Quasi 2D colloidal CdSe platelets with thicknesses controlled at the atomic level. $J$ Am Chem Soc. 2008;130 (49):16504-16505. doi:10.1021/ja807724e

2. Christodoulou S, Climente JI, Planelles J, et al. Chloride-induced thickness control in CdSe nanoplatelets. Nano Lett. 2018;18 (10):6248-6254. doi:10.1021/acs.nanolett.8b02361

3. Ithurria S, Tessier MD, Mahler B, Lobo RPSM, Dubertret B, Efros AL. Colloidal nanoplatelets with two-dimensional electronic structure. Nat Mat. 2011;10(12):936. doi:10.1038/nmat3145

4. Zelewski SJ, Nawrot KC, Zak A, Gladysiewicz M, Nyk M, Kudrawiec R. Exciton binding energy of two-dimensional highly luminescent colloidal nanostructures determined from combined optical and photoacoustic spectroscopies. J Phys Chem Lett. 2019;10 (12):3459-3464. doi:10.1021/acs.jpclett.9b00591 
5. Yang Z, Pelton M, Fedin I, Talapin DV, Waks E. A room temperature continuous-wave nanolaser using colloidal quantum wells. Nat Commun. 2017;8(1):143. doi:10.1038/s41467-017-00198-z

6. Flatten LC, Christodoulou S, Patel RK, et al. Strong exciton-photon coupling with colloidal nanoplatelets in an open microcavity. Nano Lett. 2016;16(11):7137-7141. doi:10.1021/acs.nanolett.6b03433

7. Scott R, Achtstein AW, Prudnikau A, et al. Two photon absorption in II-VI semiconductors: the influence of dimensionality and size. Nano Lett. 2015;15(8):4985-4992. doi:10.1021/acs.nanolett.5b00966

8. Won N, Jeong S, Kim K, et al. Imaging depths of near-infrared quantum dots in first and second optical windows. Mol Imaging. 2012;11(4):72. doi:10.2310/7290.2011.00057

9. Chou KL, Won N, Kwag J, Kim S, Chen JY. Femto-second laser beam with a low power density achieved a two-photon photodynamic cancer therapy with quantum dots. J Mater Chem B. 2013;1 (36):4584-4592. doi:10.1039/C3TB20928H

10. Chen N, He Y, Su Y, et al. The cytotoxicity of cadmium-based quantum dots. Biomaterials. 2012;33(5):1238-1244. doi:10.1016/j. biomaterials.2011.10.070

11. Bonilla CAM, Flórez MHT, Molina Velasco DR, Kouznetsov VV. Surface characterization of thiol ligands on CdTe quantum dots: analysis by $1 \mathrm{H}$ NMR and DOSY. New J Chem. 2019;43 (22):8452-8458. doi:10.1039/C8NJ05914D

12. Tomczak N, Liu R, Vancso JG. Polymer-coated quantum dots. Nanoscale. 2013;5(24):12018-12032. doi:10.1039/C3NR03949H

13. Yuan Q, Hein S, Misra RDK. New generation of chitosan-encapsulated $\mathrm{ZnO}$ quantum dots loaded with drug: synthesis, characterization and in vitro drug delivery response. Acta Biomat. 2010;6(7):2732-2739. doi:10.1016/j.actbio.2010.01.025

14. Edmund AR, Kambalapally S, Wilson TA, Nicolosi RJ. Encapsulation of cadmium selenide quantum dots using a self-assembling nanoemulsion (SANE) reduces their in vitro toxicity. Toxicol in Vitro. 2011;25(1):185-190. doi:10.1016/j. tiv.2010.10.017

15. Bazylińska U, Drozdek S, Nyk M, Kulbacka J, Samoć M, Wilk KA. Core/shell quantum dots encapsulated in biocompatible oil-core nanocarriers as two-photon fluorescent markers for bioimaging. Langmuir. 2014;30(49):14931-14943. doi:10.1021/la504558z

16. Kim JS, Cho KJ, Tran TH, et al. In vivo NIR imaging with CdTe/ CdSe quantum dots entrapped in PLGA nanospheres. J. Colloid Interface Sci. 2011;353(2):363-371. doi:10.1016/j.jcis.2010.08.053

17. Kodanek T, Banbela HM, Naskar S, Adel P, Bigall NC, Dorfs D. Phase transfer of 1- and 2-dimensional Cd-based nanocrystals. Nanoscale. 2015;7(45):19300-19309. doi:10.1039/C5NR06221G

18. Shendre S, Delikanli S, Li M, et al. Ultrahigh-efficiency aqueous flat nanocrystals of CdSe/CdS@Cd1-xZnxS colloidal core/crown@alloyed-shell quantum wells. Nanoscale. 2019;11(1):301-310. doi:10.1039/C8NR07879C

19. Wang X, Hao J, Cheng J, et al. Chiral CdSe nanoplatelets as an ultrasensitive probe for lead ion sensing. Nanoscale. 2019;11 (19):9327-9334. doi:10.1039/c8nr10506e
20. Nagy JA, Chang SH, Dvorak AM, Dvorak HF. Why are tumour blood vessels abnormal and why is it important to know? $\mathrm{Br}$ $J$ Cancer. 2009;100(6):865. doi:10.1038/sj.bjc.6604929

21. Fan Y, Liu H, Han R, et al. Extremely high brightness from polymer-encapsulated quantum dots for two-photon cellular and deep-tissue imaging. Sci Rep. 2015;5(1):9908. doi:10.1038/ srep09908

22. Tessier MD, Spinicelli P, Dupont D, Patriarche G, Ithurria S, Dubertret B. Efficient exciton concentrators built from colloidal core/crown $\mathrm{CdSe} / \mathrm{CdS}$ semiconductor nanoplatelets. Nano Lett. 2014;14(1):207-213. doi:10.1021/n1403746p

23. Makarov NS, Drobizhev M, Rebane A. Two-photon absorption standards in the 550-1600 nm excitation wavelength range. Opt Express. 2008;16(6):4029-4047. doi:10.1364/OE.16.004026

24. Saczko J, Dominiak M, Kulbacka J, Chwiłkowska A, Krawczykowska H. A simple and established method of tissue culture of human gingival fibroblasts for gingival augmentation. Folia Histochem. Cytobiol. 2008;46(1):117-119. doi:10.2478/v10042-0080017-4

25. Bazylinska U, Kulbacka J, Chodaczek G. Nanoemulsion structural design in co-encapsulation of hybrid multifunctional agents: influence of the smart PLGA polymers on the nanosystem-enhanced delivery and electro-photodynamic treatment. Pharmaceutics. 2019;11(8):11. doi:10.3390/pharmaceutics 11080405

26. Choi HS, Liu W, Misra P, et al. Renal clearance of quantum dots. Nat Biotechnol. 2007;25(10):1165-1170. doi:10.1038/nbt1340

27. Yetisgin AA, Cetinel S, Zuvin M, Kosar A, Kutlu O. Therapeutic nanoparticles and their targeted delivery applications. Molecules. 2020;25(9):2193. doi:10.3390/molecules25092193

28. Maddux CJA, Kelley DF, Kelley AM. Weak exciton-phonon coupling in CdSe nanoplatelets from quantitative resonance raman intensity analysis. J Phys Chem C. 2018;122(47):27100-27106. doi:10.1021/acs.jpcc.8b10125

29. Olutas M, Guzelturk B, Kelestemur Y, Yeltik A, Delikanli S, Demir HV. Lateral size-dependent spontaneous and stimulated emission properties in colloidal CdSe nanoplatelets. ACS Nano. 2015;9 (5):5041-5050. doi:10.1021/acsnano.5b01927

30. Tessier MD, Javaux C, Maksimovic I, Loriette V, Dubertret B. Spectroscopy of single CdSe nanoplatelets. ACS Nano. 2012;6 (8):6751-6758. doi:10.1021/nn3014855

31. Sekhon BS, Kamboj SR. Inorganic nanomedicine - Part 1. Nanomed Nanotechnol Biol Med. 2010;6(4):516-522. doi:10.1016/j. nano.2010.04.004

32. Ladj R, Bitar A, Eissa MM, et al. Polymer encapsulation of inorganic nanoparticles for biomedical applications. Int. J. Pharm. 2013;458 (1):230-241. doi:10.1039/C2TB00301E
International Journal of Nanomedicine

\section{Publish your work in this journal}

The International Journal of Nanomedicine is an international, peerreviewed journal focusing on the application of nanotechnology in diagnostics, therapeutics, and drug delivery systems throughout the biomedical field. This journal is indexed on PubMed Central, MedLine, CAS, SciSearch ${ }^{\mathbb{R}}$, Current Contents ${ }^{\mathbb{R}} /$ Clinical Medicine, $^{2}$
Journal Citation Reports/Science Edition, EMBase, Scopus and the Elsevier Bibliographic databases. The manuscript management system is completely online and includes a very quick and fair peer-review system, which is all easy to use. Visit http://www.dovepress.com/ testimonials.php to read real quotes from published authors. 